\title{
A New DCT based Color Video Watermarking using Luminance Component
}

\author{
Jaya Jeswani ${ }^{1}$, Dr. Tanuja Sarode ${ }^{2}$ \\ ${ }^{1}$ (Lecturer, Information Technology Department, Xavier Institute of Engineering, India) \\ ${ }^{2}$ (Associate Professor, Computer Engineering Department, Thadomal Shahani Engineering College, India)
}

\begin{abstract}
This paper presents a new blind secure video watermarking algorithm using DCT (discrete cosine transform). In this proposed algorithm cover video is divided into frames and watermark is inserted into selected frames. For selected video frames two-dimensional $8 \times 8$ discrete cosine transform is carried out on luminance component. Finally binary watermark is embedded into mid frequency DC coefficients by adjusting coefficients DCT $(4,3)$ and DCT $(5,2)$. Experimental results shows that the proposed algorithm is imperceptible as well as robust against wide variety of signal and video processing attacks like Gaussian noise, Salt -pepper noise, Gaussian filter, Median filter, Histogram Equalization etc. The technique is fairly acceptable and watermarked video is of good quality, achieves high PSNR. The technique shows efficient extraction of watermark with $N C$ value of the retrieved watermark as 1 .
\end{abstract}

Keywords: DCT (Discrete Cosine Transform), Luminance (Y component), MSE (Mean Square Error), Normalized Correlation (NC), PSNR (Peak Signal to Noise)

\section{Introduction}

Now a day's people are highly dependent on network technology, the users of networks especially over the Internet are increasing enormously. The increased importance of digital content invites new challenges for securing the distribution of digital media. This copyright misuse is the motivating factor in developing new watermarking techniques. Watermarking [1-6] can be used for copyright protection. There is a need for video watermarking $[1,2]$ as most of the information on Internet these days is in the form of videos as well. Video watermarking is a technology in which there is embedding of various copyright information in video frames [3, 6 , 7]. Digital watermarking algorithms are classified into frequency domain and spatial domain algorithms. Spatial domain algorithms embeds watermark by directly modifying pixels of carrier signal $[8,9]$ while Frequency domain algorithms embeds watermark by modifying frequency bands $[10,11]$. Frequency domain watermarking is more secure and robust as compare to spatial domain watermarking. We are developing an algorithm for video watermarking in frequency domain using DCT which embeds binary watermark in video frames. Each bit of binary watermark is embedded into different $8 \times 8$ sized DCT block of Y (Luminance) channel of selected frame. Proposed method is blind and invisible as well as robust against variety of video processing attacks.

In literature DCT transform has been successfully used for digital watermarking. In the proposed algorithm DCT is used for video watermarking in frequency domain. DCT divides carrier signal into low, middle, and high frequency bands [10]. DCT watermarking is classified into two types: Global DCT watermarking and Block-based DCT watermarking. In the Global DCT watermarking, the DCT computation is performed on the whole image, while in the Block-based DCT the image is divided into non-overlapping blocks and DCT computation is performed on each block separately to obtain low-frequency, mid-frequency and high-frequency sub-bands . J. R. Hemandez, M. Amado have proposed image watermarking in DCT domain [11]. Masoumi, M., Amiri, have Proposed video watermarking in YCbCr color space [12]. S. Feng, D. Lin, S. C. Shie and J. Y. Guo proposed a DCT-based technique they converted RGB space to YUV space and embedded watermark in $\mathrm{Y}$ component [15]. Jaya Jeswani and Tanuja Sarode have proposed a blind image watermarking using DCT in RGB color space by modifying middle frequency coefficients DCT $(4,3)$ and DCT $(5,2)[17]$.

The paper is organized as follows: Section 1 presents introduction. An introduction to DCT transform is given in section 2. Section 3 describes proposed algorithm with DCT coefficients selection, watermark embedding and extraction algorithms. Experimental results before and after applications of attacks are given in section 4. Finally conclusion of proposed algorithm is given in section 5 . 


\section{Discrete Cosine Transform}

\section{Preliminaries}

DCT(Discrete Cosine Transform)is a popular frequency domain watermarking technique [17]. DCT divides carrier signal into three frequency bands namely low, middle, and high frequency bands. It is frequency domain watermarking technique as watermark is embedded into one of these three bands, carrier signal pixels are not modified directly. Fig. 1 below shows three DCT Regions, $\mathrm{F}_{\mathrm{L}}$ is used to denote the lowest frequency components of the block, $F_{M}$ is used to denote the middle frequency components, $F_{H}$ is used to denote the higher frequency components.

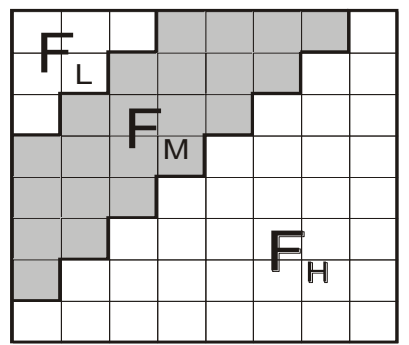

Fig. 1: DCT Regions

The definition of 2-D DCT can be given as follows:

$\mathrm{C}(\mathrm{u}, \mathrm{v})=\boldsymbol{\alpha}(\mathbf{u}) \alpha(\mathrm{v}) \sum_{x=0}^{N-1} \sum_{y=0}^{N-1} f(x, y) \cos \left[\frac{(2 x+1) u \pi}{2 N}\right] \cos \left[\frac{(2 y+1) v \pi}{2 N}\right]$ For $\mathbf{u}, \mathbf{v}=\mathbf{0}, 1,2, \ldots, \mathbf{N}-1$

Above equation 1 converts image from spatial domain to frequency domain,

Image can be transformed back to spatial domain by applying inverse DCT given in equation 2:

The definition of 2-D inverse DCT can be written as follows:

$f(\mathbf{x}, \mathbf{y})=\sum_{u=0}^{N-1} \sum_{v=0}^{N-1} \alpha(\mathbf{u}) \alpha(v) C(u, v) \cos \left[\frac{(2 x+1) u \pi}{2 N}\right] \cos \left[\frac{(2 y+1) v \pi}{2 N}\right] \quad$ For $\mathbf{x}, \mathbf{y}=0,1,2, \ldots, \mathbf{N}-1$

$\alpha(\mathbf{u}), \alpha(\mathbf{v})=$

$$
\sqrt{1 / \mathrm{N}} \quad \text { for } \mathrm{u}, \mathrm{v}=\mathbf{0}
$$

$$
\sqrt{2 / N} \quad \text { for } u, v=1,2, \ldots . ., N-1
$$

In this paper for watermark embedding middle frequency bands are selected because moreof the video energy lies on low-frequency sub-band which contains the most important visual contents of video which effects quality of watermarked video, high frequency sub-band is usually removed through noise attacks.

\section{Proposed Method}

Proposed method is divided into three subsections section A explains DCT Coefficients Selection section $\mathrm{B}$ describes watermark insertion process and section $\mathrm{C}$ describes watermark extraction process.

\section{A. DCT Coefficients Selection}

For watermark embedding DCT coefficients DCT $(4,3)$ and DCT $(5,2)$ have been selected because both are middle frequency components and in JPEG quantization table both are having same value as 22 . The choice in selecting the two locations is dependent on the content of the JPEG quantization table given below in table I.

Table I: JPEG Quantization Table

\begin{tabular}{|l|l|l|l|l|l|l|l|}
\hline 16 & 11 & 10 & 26 & 24 & 40 & 51 & 61 \\
\hline 12 & 12 & 14 & 19 & 26 & 58 & 60 & 55 \\
\hline 14 & 13 & 16 & 24 & 40 & 57 & 69 & 56 \\
\hline 14 & 17 & 22 & 29 & 51 & 87 & 80 & 62 \\
\hline 18 & $\mathbf{2 2}$ & 37 & 56 & 68 & 109 & 103 & 77 \\
\hline 24 & 35 & 55 & 64 & 81 & 104 & 113 & 92 \\
\hline 49 & 64 & 78 & 87 & 103 & 121 & 120 & 101 \\
\hline 72 & 92 & 95 & 98 & 112 & 100 & 103 & 99 \\
\hline
\end{tabular}

\section{B. Watermark Embedding Process}

Inputs: Color video frames and binary watermark

Outputs: Watermarked video frames 


\section{The steps are as follows:}

1. Take cover video of size $\mathrm{M} \times \mathrm{N}$ and select some video frames where watermark is to be embedded. Binary watermarkof size $\mathrm{n} \times \mathrm{n}$ is also taken as an input.

2. $\quad$ Selected frames are decompose into 3 components: $\mathrm{Y}, \mathrm{U}$ and $\mathrm{V}$.

3. Select Luminance component for watermark embedding and divide it into $8 \times 8$ sized blocks.

4. Determine watermark size based on cover image and block size by :

watermark_size $=\mathbf{M} \times \mathbf{N} / \mathbf{b l o c k}$ size $^{2}$

5. Check watermark size if it is less than the watermark size calculated by equation 4 than pad the watermark out to the watermark size with ones.

6. Transform each block using DCT.

7. Embeds watermark bit $=0$ when DCT $(5,2)$ is greater than or equal to $\mathrm{DCT}(4,3)$ and embeds watermark bit=1 when DCT $(5,2)$ is less than DCT $(4,3)$.

8. If watermark bit $=0$, then $\operatorname{DCT}(5,2)$ should be greater than or equals to $\operatorname{DCT}(4,3)$ and if $\operatorname{DCT}(5,2)$ less than $\operatorname{DCT}(4,3)$ then swap these two values .

9. If watermark bit=1, then $\operatorname{DCT}(5,2)$ should be less than DCT $(4,3)$ and if $\operatorname{DCT}(5,2)$ greater DCT $(4,3)$ then swap these two values.

10. Adjust difference between $\operatorname{DCT}(5,2)$ and $\operatorname{DCT}(4,3)$ such that their difference $=\mathrm{k}$.

11. Transform block back into spatial domain by IDCT which gives watermarked frame.

12. Combine modified $\mathrm{Y}$ and $\mathrm{U}, \mathrm{V}$ components to create watermarked video frame.

13. Repeat the same procedure till all the selected frames are watermarked.

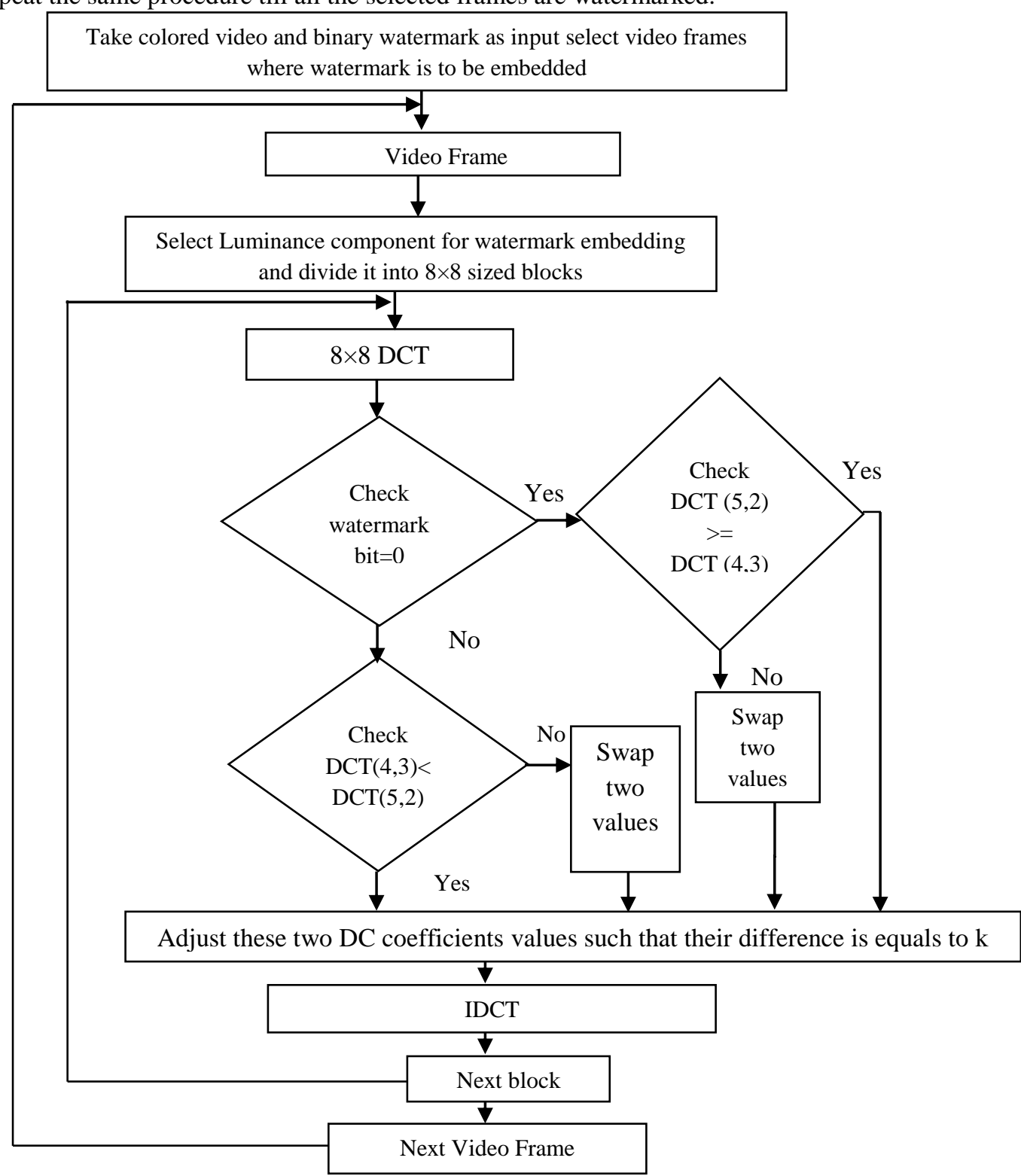

Fig.3: Flow Chart of Watermark Extraction Process 


\section{Watermark Extraction Process}

Input: Watermarked Video Frames

Output: Binary watermarks extracted from all Watermarked Frames

Steps:

1. Take watermarked video frames of size $\mathrm{M} \times \mathrm{N}$ as an input.

2. Each watermarked video frame and decompose into 3 components: $\mathrm{Y}, \mathrm{U}$ and $\mathrm{V}$.

3. Two-dimensional $8 \times 8$ discrete cosine transform is carried out on luminance component.

4. If DCT $(5,2)$ greater than or equal to coefficient of DCT $(4,3)$, make watermark bit=0 else watermark bit $=1$.

5. Reshape the recovered watermark image into $n \times n$.

6. Repeat the procedure till all the watermarks are extracted from the watermarked video frames.

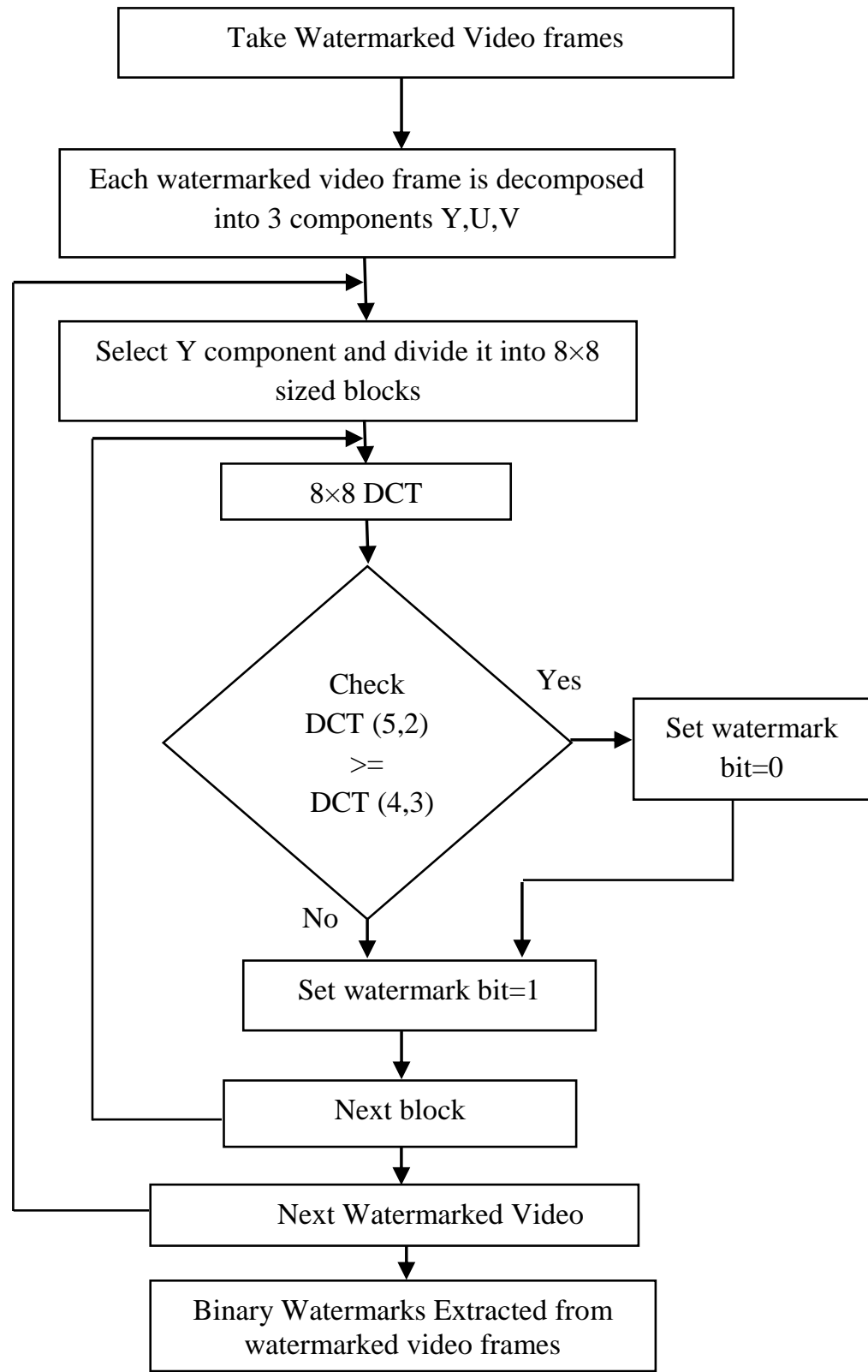

Fig.3: Flow Chart of Watermark Extraction Process

Fig.2 and Fig. 3 gives flow chart of watermark embedding and extracting process respectively. 


\section{Experimental Results}

The proposed video watermarking algorithm is implemented on Intel Core i5-3210M, $1.8 \mathrm{GHz}, 4 \mathrm{~GB}$ RAM machine and Matlab R2011b.The proposed method is tested on different videos like News, Ice, Crew, Soccer of size $256 \times 256$ and binary watermark (8.bmp) of size $32 \times 32$ is used as watermark. For evaluating the performance of proposed algorithm Peak Signal to Noise Ratio (PSNR), Mean Square Error (MSE) and Normalized correlation (NC) performance evaluators are used.

$\mathrm{MSE}=\frac{1}{\mathrm{MN}} \sum_{\mathrm{i}=1}^{\mathrm{M}} \sum_{\mathrm{j}=1}^{\mathrm{N}}\left[\mathrm{I}(\mathbf{i}, \mathbf{j})-\mathrm{I}^{\prime}(\mathbf{i}, \mathbf{j})\right]^{2}$

PSNR $=10 \log _{10}\left(\frac{255^{2}}{\text { MSE }}\right)$

Where, $\mathrm{M}, \mathrm{N}=$ size of the original video frame,

$I(i, j)=$ pixel values at location $(i, j)$ of the original video frame,

$I^{\prime}(i, j)=$ pixel values at location $(i, j)$ of watermarked video frame

$\mathrm{NC}=\frac{\sum_{i} \sum_{j} \boldsymbol{w}(i, j) w r(i, j)}{\sum_{i} \sum_{j} \boldsymbol{w}(i, j)^{2}}$

$W(i, j)=$ pixel values at location $(i, j)$ of the original watermark,

$W^{\prime}(i, j)=$ pixel values at location $(i, j)$ of the extracted watermark

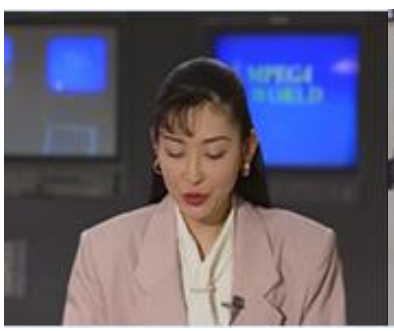

(a)

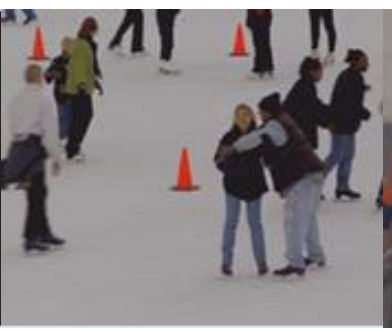

(b)

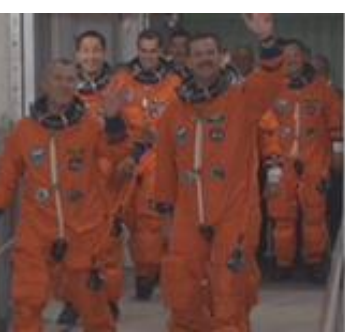

(c)

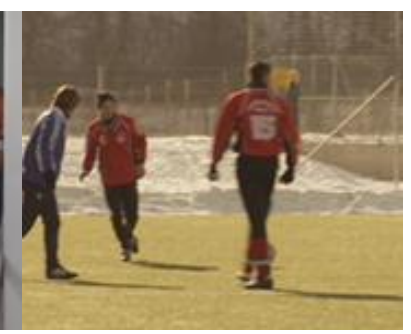

(d)

Fig. 4 Cover Videos (a) News (b) Ice (c) Crew (d) Soccer

Table II: PSNR values of first nine watermarked frames of the News.y4m video at different values of $\mathrm{k}$

\begin{tabular}{|c|c|c|c|c|c|c|c|c|c|c|}
\hline $\begin{array}{c}\text { Quality } \\
\text { Factor } \\
\text { K }\end{array}$ & \multicolumn{9}{|c|}{ PSNR } \\
\hline 1 & 45.3948 & 46.3186 & 46.3031 & 46.2931 & 46.2757 & 46.2800 & 46.2956 & 46.2009 & 46.1932 & 46.1745 \\
\hline 2 & 45.3635 & 46.2812 & 46.2674 & 46.2568 & 46.2459 & 46.2478 & 46.2529 & 46.1664 & 46.1672 & 46.1388 \\
\hline 3 & 45.3108 & 46.2112 & 46.1992 & 46.1906 & 46.1806 & 46.1860 & 46.1788 & 46.1006 & 46.0942 & 46.0725 \\
\hline 4 & 45.2826 & 46.1478 & 46.1346 & 46.1273 & 46.1077 & 46.1257 & 46.1273 & 46.0465 & 46.0302 & 46.0144 \\
\hline $\mathbf{5}$ & 45.2291 & 46.0679 & 46.0665 & 46.0364 & 46.0269 & 46.0403 & 46.0329 & 45.9669 & 45.9609 & $\mathbf{4 5 . 9 3 6 4}$ \\
\hline 6 & 45.1703 & 45.9814 & 45.9848 & 45.9757 & 45.9453 & 45.9640 & 45.9587 & 45.8901 & 45.8990 & 45.8632 \\
\hline 7 & 45.1152 & 45.9230 & 45.9220 & 45.9063 & 45.8831 & 45.8974 & 45.8949 & 45.8319 & 45.8294 & 45.8003 \\
\hline 8 & 45.0448 & 45.8604 & 45.8551 & 45.8477 & 45.8090 & 45.8330 & 45.8387 & 45.7710 & 45.7679 & 45.7364 \\
\hline 9 & 44.9915 & 45.8083 & 45.7942 & 45.7796 & 45.7570 & 45.7879 & 45.7858 & 45.7143 & 45.7066 & 45.6805 \\
\hline 10 & 44.9367 & 45.7496 & 45.7196 & 45.7164 & 45.6903 & 45.7000 & 45.7162 & 45.6542 & 45.6449 & 45.6102 \\
\hline
\end{tabular}

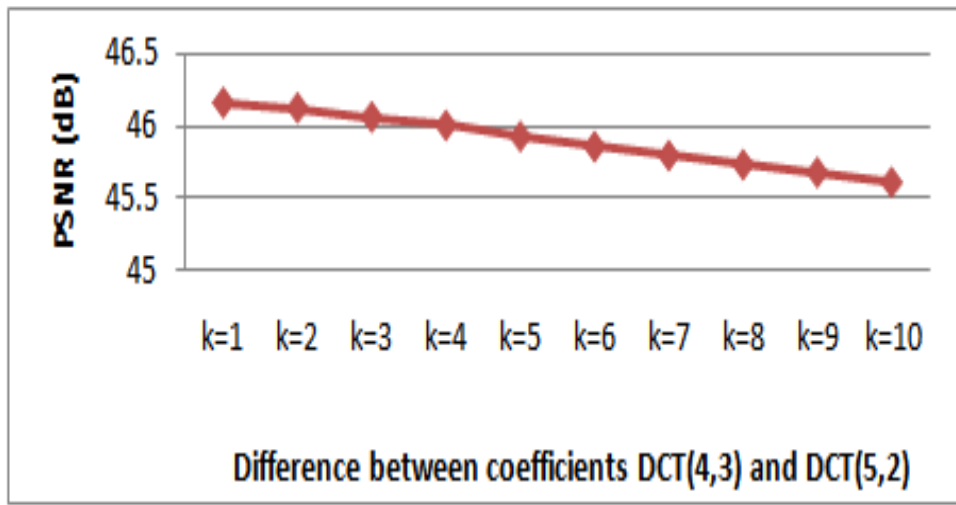

Fig.5:Peak Signal to Noise Ratio Chart at different Values of $k$

From above table II it can be concluded that as the value of $\mathrm{k}$ increases PSNR of watermarked frames decreases. Fig.5 shows Peak Signal to Noise Ratio chart at different values of k. Here k indicates minimum 
coefficient difference between DCT(4,3) and DCT(5,2).Fig. 6 below shows results of watermark embedding using proposed algorithm on $1^{\text {st }}$ frame and $48^{\text {th }}$ frame respectively. Original watermark and extracted watermark from $1^{\text {st }}$ frame and $48^{\text {th }}$ frame respectively are shown in Fig. 7.First 48 frames of video News.y $4 \mathrm{~m}$ are selected for watermark embedding and Table III, Table IV shows PSNR and MSE values of first 48 watermarked frames respectively at $\mathrm{k}=5$. Value of $\mathrm{k}$ selected is 5 because it gives optimum performance and after attack also watermark is extracted with some distortion. Fig. 8 shows PSNR and MSE charts of 48 watermarked frames.

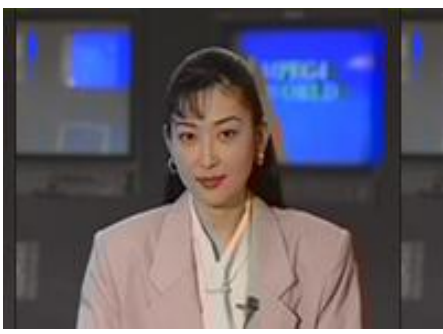

(a)

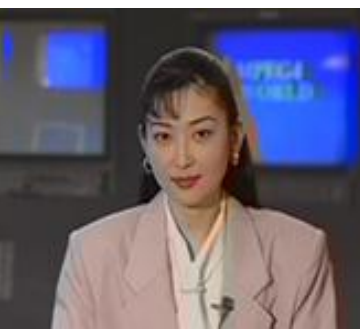

(b)

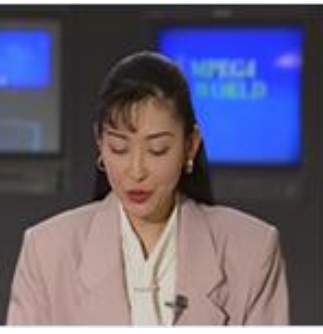

(c)

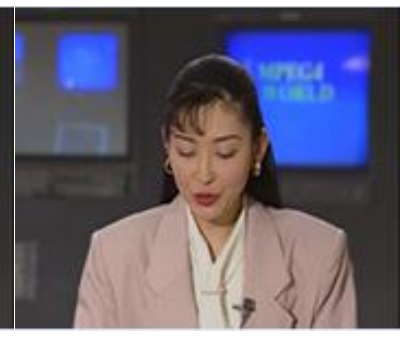

(d)

Fig.6 (a) $1^{\text {st }}$ frame of News.y4m video (b) $1^{\text {st }}$ Watermarked frame of News.y4m video(c) $48^{\text {th }}$ frame of News.y4m video (d) $48^{\text {th }}$ Watermarked frame of News.y $4 \mathrm{~m}$ video

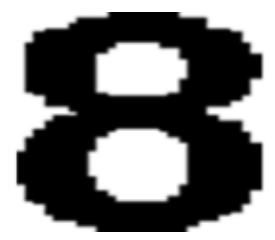

(a)

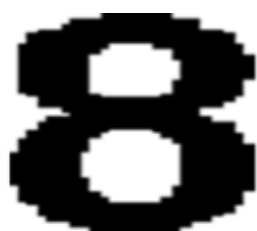

(b)

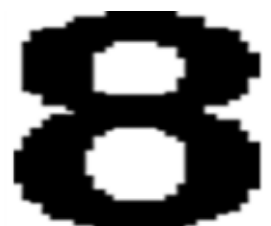

(c)

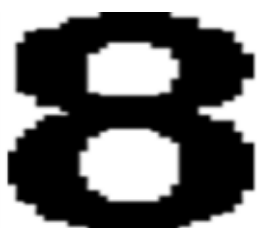

(d)

Fig.7(a) Original Watermark(b) Watermark extracted from $1^{\text {st }}$ frame of News.y4m video(c) Original Watermark(d) Watermark extracted from $48^{\text {th }}$ frame of News.y $4 \mathrm{~m}$ video

Table III: PSNR values of first 48 watermarked frames of the News.y4m Video

\begin{tabular}{|c|c|c|c|c|c|c|c|c|}
\hline Frames & \multicolumn{7}{|c|}{ PSNR at k=5 } \\
\hline $1-8$ & 45.2291 & 46.0679 & 46.0665 & 46.0364 & 46.0269 & 46.0403 & 46.0329 & 45.9669 \\
\hline $9-16$ & 45.9606 & 46.0241 & 46.0479 & 46.1100 & 46.0572 & 46.0245 & 46.1043 & 46.0634 \\
\hline $17-24$ & 45.8748 & 45.9035 & 45.9653 & 45.9518 & 45.9331 & 45.9861 & 46.0025 & 46.0039 \\
\hline $25-32$ & 45.9223 & 45.9933 & 46.0465 & 46.0135 & 46.1171 & 45.9975 & 45.8599 & 45.9712 \\
\hline $26-40$ & 46.0661 & 46.0263 & 46.0107 & 46.0488 & 46.1461 & 46.1133 & 45.9831 & 46.0650 \\
\hline $41-48$ & 46.1544 & 46.1535 & 46.1162 & 46.1006 & 46.0290 & 46.0372 & 46.0956 & 46.1010 \\
\hline Average & \multicolumn{70}{|c|}{$\mathbf{4 6 . 0 1 3 5}(\mathbf{d B})$} \\
\hline
\end{tabular}

Table IV: MSE values of first 48 watermarked frames of the News.y4m Video

\begin{tabular}{|c|c|c|c|c|c|c|c|c|}
\hline Frames & \multicolumn{7}{|c|}{ MSE at k=5 } \\
\hline $1-8$ & 1.9659 & 1.6207 & 1.6212 & 1.6325 & 1.6360 & 1.6310 & 1.6338 & 1.6588 \\
\hline $9-16$ & 1.6612 & 1.6371 & 1.6281 & 1.6050 & 1.6246 & 1.6369 & 1.6071 & 1.6223 \\
\hline $17-24$ & 1.6943 & 1.6832 & 1.6594 & 1.6646 & 1.6718 & 1.6515 & 1.6452 & 1.6447 \\
\hline $25-32$ & 1.6759 & 1.6487 & 1.6287 & 1.6411 & 1.6024 & 1.6471 & 1.7002 & 1.6572 \\
\hline $26-40$ & 1.6213 & 1.6362 & 1.6421 & 1.6278 & 1.5917 & 1.6038 & 1.6526 & 1.6217 \\
\hline $41-48$ & 1.5887 & 1.5890 & 1.6027 & 1.6085 & 1.6352 & 1.6321 & 1.6104 & 1.6083 \\
\hline Average & \multicolumn{7}{|c|}{$\mathbf{1 . 6 4 2 5}(\mathbf{d B})$} \\
\hline
\end{tabular}

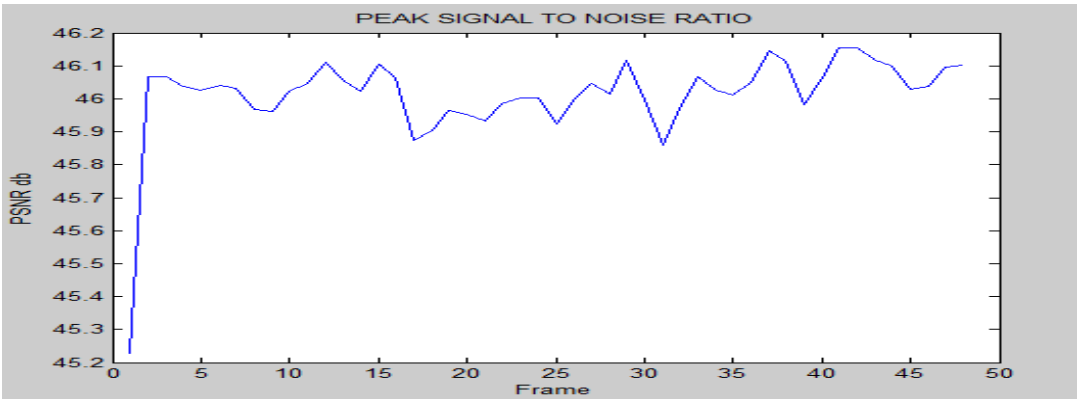

(a) 


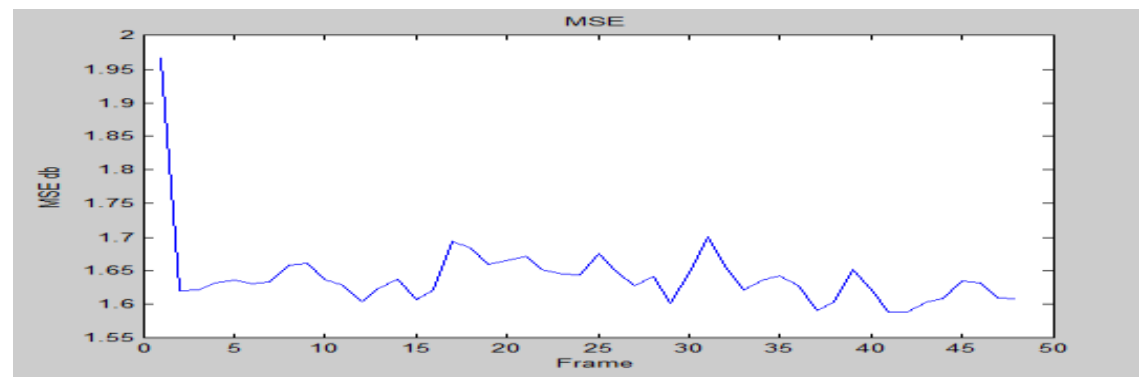

(b)

Fig.8: (a) Peak Signal to Noise Ratio Chart (b) Mean Square Error chart

Table V. Experimental results of common signal and video processing attacks on News.y4m video

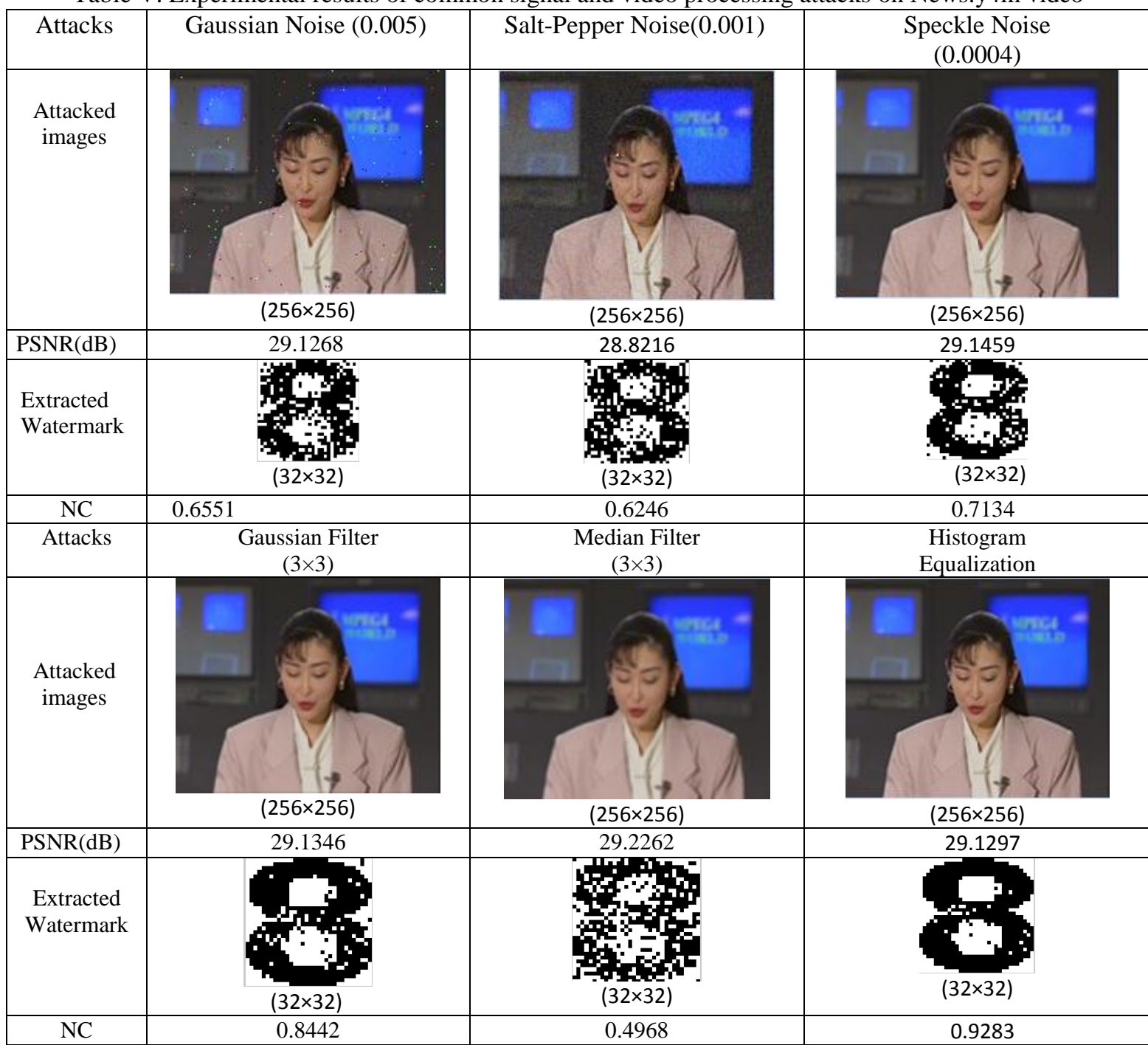

Table VI: Results of video watermarking on different test cover videos

\begin{tabular}{|c|c|c|c|}
\hline $\begin{array}{c}\text { Test Carrier } \\
\text { Videos }\end{array}$ & $\begin{array}{c}\text { Average PSNR of } \\
\text { Watermarked Frames }\end{array}$ & $\begin{array}{c}\text { MSE of Watermarked } \\
\text { Frames }\end{array}$ & $\begin{array}{c}\text { Average NC of } \\
\text { Extracted watermarks }\end{array}$ \\
\hline News & 46.0135 & 1.6425 & 1 \\
\hline Ice & 45.6489 & 1.7848 & 1 \\
\hline Crew & 46.6249 & 1.4256 & 1 \\
\hline Soccer & 46.5829 & 1.4394 & 1 \\
\hline
\end{tabular}


Results of various attacks are shown in Table V with PSNR and NC values after application of attacks. Table 4 below shows results of watermarking on different test videos namely NEWS, ICE, CREW, SOCCER. It shows average PSNR of watermarked video along with MSE and NC value of extracted watermarks. It can be seen that in all the different test videos PSNR achieved is high and NC value is 1 means extracted watermark is same as that of original watermark.

\section{Conclusion}

Various video watermarking algorithms have been proposed in spatial and frequency domains but very few algorithms are proposed for color videos. In this paper a frequency domain color video watermarking is proposed by using DCT. Proposed algorithm is blind video watermarking algorithm so at the time of watermark extraction original video frames are not required and recovery of watermark is lossless and having NC value as 1 without any attack. Proposed method is robust and secure because watermark is inserted in only Y component and chrominance component that is $\mathrm{u}$ and $\mathrm{v}$ are untouched. The proposed method is fairly acceptable and can be used as a non-blind video watermarking algorithm. The performance of proposed algorithm is measured by computing the Peak Signal to Noise Ratio (PSNR) and Normalized correlation (NC). The proposed method achieves average PSNR as $45.98 \mathrm{~dB}$ and $\mathrm{NC}$ as 1. Experimental results show that proposed method is imperceptible as well as robust against variety of attacks like salt- pepper noise, Gaussian noise, Speckle noise and filtering attacks like Median filter, Gaussian filter etc. Thus, proposed can be used as a non-blind video watermarking algorithm.

\section{References}

[1] C.I. Podilchuk, E.J. Delp “Digital watermarking: algorithms and applications,” Signal Processing Magazine, IEEE,Vol 18, pp.33-46 July 2001.

[2] Potdar, V. M., Han, S., and Chang, E., "A Survey of Digital Image Watermarking Techniques", 3rd IEEE International Conference on Industrial Informatics (INDIN), pp. 709-716, 2005.

[3] Yeo and M.M. Yeung, "Analysis and synthesis for new digital video applications", International Conference on Image Processing (ICIP'97), vol. 1, pp.1, 1997.

[4] SmithaRao, Jyothsna A. N, PinakaPani. R, "Digital watermarking: applications, techniques and attacks", International Journal of Computer Applications Volume 44, No. 7, pp. 29-34, April 2012.

[5] M. Natarajan, G. Makhdumi1, "Safeguarding the Digital Contents: Digital Watermarking," DESIDOC Journal of Library \&Information Technology, Vol. 29, pp. 29-35, May 2009.

[6] G. Doërr, J.L. Dugelay, "Security Pitfalls of Frame-by-Frame Approaches to Video Watermarking," Signal Processing, IEEE Transactions, vol. 52, pp. $2955-2964,2004$.

[7] M. K. Thakur, V. Saxena, J. P.Gupta, "A Performance Analysis of Objective Video Quality Metrics for Digital Video Watermarking," Computer Science and Information Technology (ICCSIT), 2010, 3rd IEEE International Conference, Vol. 4, pp. 12- 17, 2010.

[8] ManikMondal, DebalinaBarik,. "Spatial domain robust watermarking scheme for color image," International Journal of Advanced Computer Science, vol.2, no.1,pp. 24-27, Jan 2012.

[9] T. Tokar, T. Kanocz, D. Levicky, "Digital watermarking of uncompressed video in spatial domain," 9th International Conference on Radioelectronica, IEEE, pp. 319-322, 2009.

[10] Rao K, P. Yip, "Discrete Cosine Transform: algorithms, advantages, applications", Academic press, USA, 1990.

[11] J. R. Hemandez, M. Amado, "DCT domain watermarking techniques for still images as detector performance analysis and a new structure," in IEEE Transactions on Image Processing, vol. 9, pp. 55-68,2000.

[12] Masoumi, M., Amiri, S., "A High Capacity Digital Watermarking Scheme for Copyright protection of Video Data based on YCbCr Color Channels Invariant to Geometric and Non-Geometric Attacks", International Journal of Computer Applications, vol.51, no.13, pp. 0975-8887, August 2012.

[13] R. Eswaraiah\& E. Sreenivas a Reddy, "Robust Watermarking Method for Color Images Using DCT Coefficients of Watermark", Global Journals Inc(US) 2012.

[14] Dr. K. Ramanjaneyulu, Dr. P. Pandarinath and B. Rakesh Reddy, "Robust and Oblivious Watermarking based on Swapping of DCT Coefficients", International Journal of Application or Innovation in Engineering \& Management, vol. 2, issue 7, July 2013.

[15] Lu Jianfeng; Yang Zhenhua; Yang Fan; Li li “A MPEG2 Video Watermarking Algorithm Based on DCT Domain" Institute of Computer Graphics and Image Processing IEEE Computer society ,2011.

[16] S. Feng, D. Lin, S. C. Shie, J. Y. Guo, "Improving the robustness of DCT based image watermarking against JPEG compression", Computer Standards \& Interface, vol. 32, pp. 54-60, 2010.

[17] Jaya Jeswani, Dr. Tanuja Sarode, "An Improved Blind Color Image Watermarking Using DCT in RGB Color Space”, International Journal of Computer Applications (IJCA), Vol 92, No. 14, april 2014. 